\title{
Alignment of Self-Assembled Hierarchical Microstructure in Liquid Crystalline Diblock Copolymers Using High Magnetic Fields
}

\author{
Chinedum Osuji, ${ }^{\dagger}$ Paulo J. Ferreira, ${ }^{\S}$ Guoping Mao, ${ }^{\ddagger}$ Christopher K. Ober, ${ }^{\perp}$ \\ John B. Vander Sande, ${ }^{\dagger}$ and Edwin L. Thomas ${ }^{*, \dagger}$ \\ Program in Polymer Science and Technology, Department of Materials Science and Engineering, \\ Massachusetts Institute of Technology, Cambridge Massachusetts 02139; 3M Corporation, \\ Minneapolis, Minnesota 55125; Department of Materials Science and Engineering, University of Texas, \\ Austin, Texas 78712; and Department of Materials Science and Engineering, Cornell University, \\ Ithaca, New York 14850
}

Received August 17, 2004; Revised Manuscript Received October 8, 2004

\begin{abstract}
Large area microdomain alignment in a ferroelectric liquid crystalline diblock copolymer (LCBCP) poly(styrene)-block-poly(isoprene-LC), (PS-PILC), incorporating a biphenyl 3-nitro-4-alkoxybenzoate LC mesogenic group and a non-LC block hexagonally packed cylinder microstructure, was successfully accomplished by application of a magnetic field at elevated temperatures. Small- and mediumangle X-ray scattering demonstrated that the PS cylinders in the LC matrix orient over large areas with their long axes perpendicular to the applied magnetic field. Correspondingly, the smectic layers of the LC mesophase in the matrix are also perpendicular to the field as the anchoring of the mesogens at the intermaterial dividing surface (IMDS) between the cylindrical microdomains and the matrix is planar (homogeneous) in this material. A negative diamagnetic anisotropy for the LC mesogens is inferred from the data. A lamellar sample was also studied and found to exhibit no preferred microstructural orientation when subjected to the magnetic field. This result is consistent with the orientational state degeneracy of planar anchoring of mesogens at the flat lamellar IMDS and closely parallels our prior results obtained by orientation of nonferroelectric LCBCPs using oscillatory shear.
\end{abstract}

\section{Introduction}

Increasingly, research on the structure of soft matter has focused not just on the physics of the self-assembly of interesting materials, but on methods to control and direct the formation of the structures themselves, whether by understanding and directly manipulating the physics of self-assembly in the materials ${ }^{1-4}$ or simply by empirically searching for useful process conditions. Structure-property-processing relationships in novel and emerging classes of soft materials are, more and more, highlighted by a comprehensive understanding of structure on the micro- and nanoscales.

Block copolymers, while not entirely new and unknown materials, still do represent one such class of soft matter-many demonstrate great promise as materials for application in various macroscopic as well as microscopic technologies, ${ }^{5}$ and their performance is often greatly affected by both their rich structure on the micron scale and at the microdomain level. Thus, methods are sought to control and produce globally wellaligned microstructure in these materials. Liquid crystalline block copolymers incorporate the mesoscale selfassembly of mesogenic units within the broader context of microphase separation and block microdomain formation. The interplay of the two self-assembly processes and the consequences thereof on the structure-property relationships in these materials has been well studied by other workers in this field, ${ }^{6-10}$ including work on mechanical shear alignment. ${ }^{11,12}$

\footnotetext{
Massachusetts Institute of Technology.

$\doteqdot 3 \mathrm{M}$ Corporation.

$\S$ University of Texas.

$\perp$ Cornell University.

* To whom correspondence should be addressed.
}

In non-liquid-crystalline systems, direct application of mechanical force by both shear flow and tensile elongation, for example, ${ }^{13-15}$ has been used to orient block copolymers in the bulk state. More recently, electric fields ${ }^{16}$ as well as surface epitaxy ${ }^{17,18}$ and directional solidification ${ }^{19,20}$ in appropriate systems have been used to provide alignment of the microdomains over large areas in thin films. PTFE has been used successfully to orient smectic layers in liquid crystalline homopolymers, ${ }^{21}$ though it is not known whether the mechanism is strictly epitaxial or grapho-epitaxial-the smectic layers aligned parallel to the PTFE chain axis due to the mesogens adopting the $5.6 \AA$ interchain spacing offered by the PTFE (100) contact surface. PTFE monolayers have also been used to orient small molecules ${ }^{22}$ some of which can then be polymerized at the surface to form oriented polymers. ${ }^{23}$ For different applications, each alignment method has it advantages and disadvantages. For example, mechanical manipulation might be favored over surface epitaxy for the production of well-aligned microstructures in a material intended for bulk structural applications, but perhaps not in the fabrication of a thin film of active material (less than $10 \mu \mathrm{m}$ ) to be used in some device. In the particular case of electrooptic device fabrication, "clean" and/or "tidy" methods are sought for producing alignment in the material.

Magnetic fields are a good candidate here due to their space pervasive nature and the ability to apply large fields under different atmospheric conditions (saturated solvent conditions, for example) without the danger of the dielectric breakdown that may accompany the use of high electric fields. Recently, ${ }^{24}$ Hamley et al. found that application of a magnetic field during the isotropicsmectic transition in a side group liquid crystal polymer 


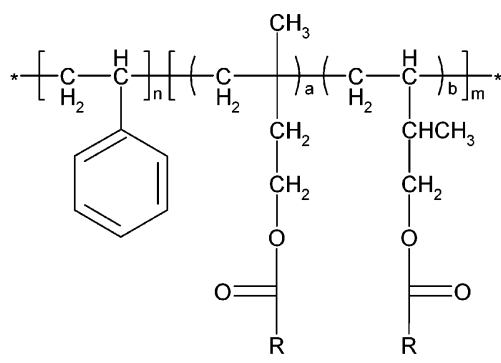<smiles>CCCCCCC(C)Oc1ccc(C(=O)Oc2ccc(-c3ccc(OCCC)cc3)cc2)cc1[N+](=O)[O-]</smiles>

Figure 1. Chemical structure of the PS-PILC diblock copolymers.

resulted in alignment for a sample consisting of hexagonally packed LC cylinders in a PS matrix but not for the inverse morphology of PS cylinders in the LC matrix. Using SANS, they observed that the LC cylinder microdomains were perpendicular to the direction of the applied field, and they inferred a homeotropic anchoring of the mesogens at the IMDS such that the smectic layers were parallel to the cylinders. Implicit is that the mesogens tend to align along the direction of the field. Such an arrangement is in fact degenerate as there are an infinite number of orientations that a cylinder can assume perpendicular to any given magnetic field direction. Further, if the anchoring of the mesogens is homeotropic such that the cylindrical microdomains are composed of concentric rings of smectic layers parallel to the cylinder as they suggest, there should be no net anisotropy in the diamagnetic susceptibility of the microdomains along the long axis of the mesogen or the layer normal-the director orientation is not unique. It is thus somewhat unclear as to what the driving force for the observed orientation was. Unfortunately, they were unable to directly observe the orientation of their smectic layers using SANS. It is possible that the boundary condition for the mesogens in this system is quite soft and that the mesogens are able to adopt both homogeneous and hometropic anchoring at the IMDS. However, in such a case, one would then expect that they would all lie homogeneous to the interface and that the cylinders would then orient along the field direction.

The driving force for the magnetic field alignment of typical liquid crystalline mesophases is the collective anisotropy of the mesophase diamagnetic susceptibility, $\chi_{\alpha}=\chi_{\|}-\chi_{\perp}$, with respect to the long axis direction of the LC mesogen. This anisotropy provides for a free energy difference between the randomly oriented state and the parallel or perpendicular orientation state of the mesophase. If this difference is significant with respect to $k T$, the thermal energy available to the system, then the mesophase will align as prescribed by the anisotropy. An expression for the excess free energy of a system with magnetization $\mathbf{M}$ in a field $\mathbf{H}$ is given in eq 1. Minimization of this excess energy occurs by parallel alignment of the mesophase director $n$ with the field direction $\vec{H}$ for mesophases with positive anisotropy $\chi_{\alpha}>0$ and by perpendicular alignment for those with

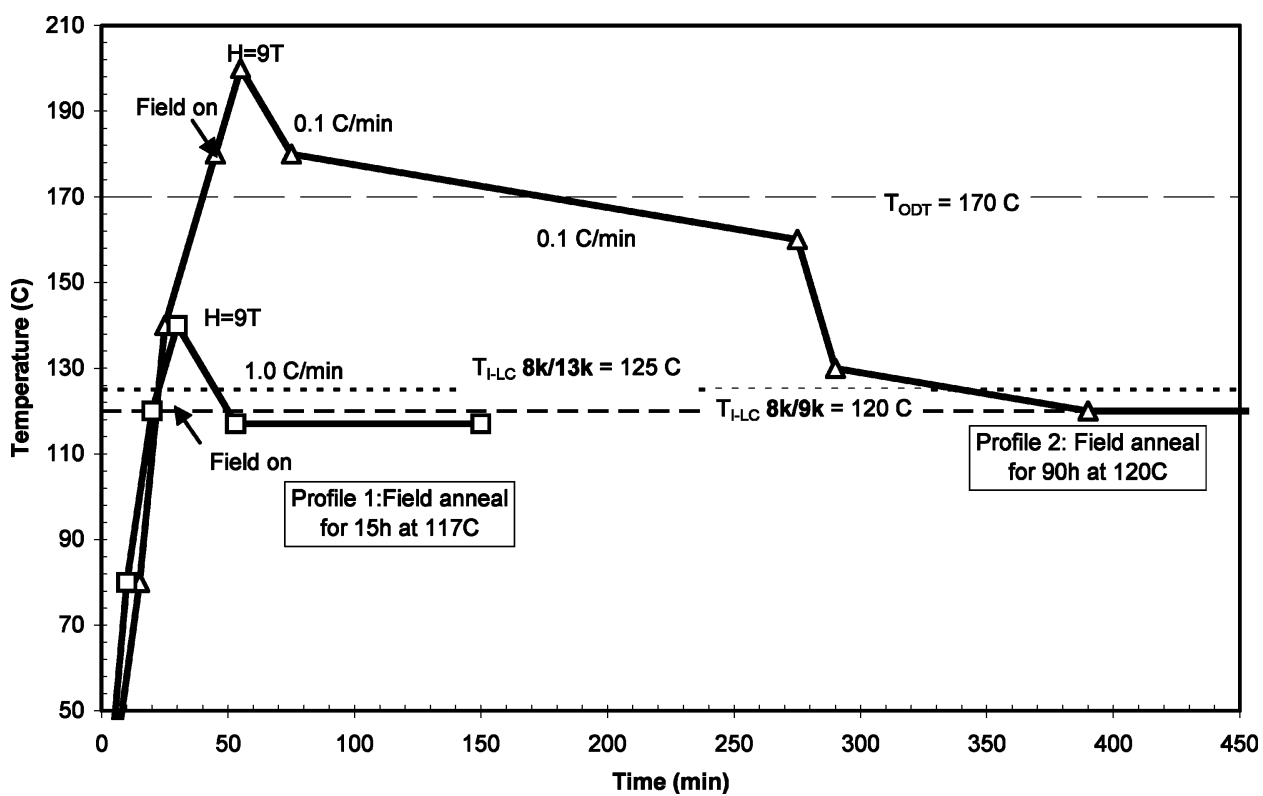

Figure 2. Temperature profiles used. The points at which the field is turned on and at which it reaches final strength are shown. Cooling rates are indicated starting from the point at which the samples are at their highest temperatures and under full field strength to the point at which they reach the experimental annealing conditions.

Table 1

\begin{tabular}{|c|c|c|c|c|c|c|}
\hline PS-PILC & $\Phi_{\mathrm{LC}}(\%)$ & $M_{\mathrm{w}}(\mathrm{kg} / \mathrm{mol})$ & PDI & thermal data & $\Delta H(\mathrm{~J} / \mathrm{g})$ normalized & morphology \\
\hline $8 \mathrm{~K}-13 \mathrm{~K}$ & 62 & 21 & 1.08 & 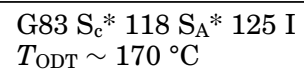 & 2.58 & $\begin{array}{l}\text { PS cylinders } \\
d_{1010}=14 \mathrm{~nm}\end{array}$ \\
\hline $8 \mathrm{~K}-9 \mathrm{~K}$ & 53 & 17 & 1.08 & 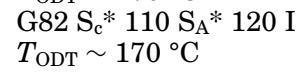 & 1.89 & $\begin{array}{l}\text { lamellae } \\
d_{001}=12 \mathrm{~nm}\end{array}$ \\
\hline \multicolumn{2}{|c|}{ PS-PB-PS } & $\Phi_{\mathrm{PS}}(\%)$ & \multicolumn{2}{|c|}{$M_{\mathrm{w}}(\mathrm{kg} / \mathrm{mol})$} & \multicolumn{2}{|c|}{ morphology } \\
\hline & & 43 & \multicolumn{2}{|c|}{58} & \multicolumn{2}{|c|}{ lamellae $d_{001}=28 \mathrm{~nm}$} \\
\hline
\end{tabular}


negative anisotropy $\chi_{\alpha}<0$.

$$
F_{\overrightarrow{\mathrm{H}}}^{\mathrm{excess}}=-\int_{0}^{H} \vec{M} \cdot \mathrm{d} \vec{H}=-\frac{1}{2} \chi_{\perp} H^{2}-\frac{1}{2} \chi_{\alpha}(\vec{n} \cdot \vec{H})^{2}
$$

Here we report on the alignment of ferroelectric liquid crystalline block copolymers using a magnetic field. These materials have a demonstrated ability to function as monostable electrooptic switches and, more favorably, as bistable switches, when oriented by simple shearing between glass slides. ${ }^{25}$ The degree of alignment obtained by shearing is limited and does not readily afford the reproducible creation of large areas with uniform orientation of microstructure. Magnetic field alignment was thus pursued as a more appropriate means of microstructure control.

\section{Experimental Section}

The primary materials under investigation were two of a series of poly(styrene)-block-poly(isoprene-LC) block copolymers, PS-PILC, synthesized using anionic polymerization followed by attachment of LC mesogens to the isoprene block as described elsewhere. ${ }^{26}$ The chemical structure of the diblocks is shown in Figure 1, and the molecular weights and compositions are given in Table 1 . Morphologies of the bulk samples were previously determined using transmission electron microscopy (TEM) and small-angle X-ray scattering (SAXS). Samples were prepared by room temperature evaporation of solvent from a 5 wt \% solution of the copolymer in toluene. Small pieces (roughly $4 \times 4 \mathrm{~mm}, 1 \mathrm{~mm}$ thick) of the copolymers were taken from the solvent-cast films and individually loosely encased in Teflon tape and stacked vertically in a small ceramic crucible. Similarly prepared samples of a commercial lamellar forming poly(styrene)-block-poly(butadiene)-block-poly(styrene) triblock copolymer, PS-PB-PS, with molecular weight of $58 \mathrm{~kg} / \mathrm{mol}$ were included as a control experiment. The crucible was placed in a sealed glass tube that permitted control over the atmosphere around the sample. The tube was lowered into the constant field core region of an Oxford Instruments superconducting magnet (ultimate flux density $=9 \mathrm{~T}$ or $9 \mathrm{~Wb} / \mathrm{m}^{2}$ ), and the tube evacuated to $10^{-5}$ Torr. Two temperature-field profiles were used. Under the first profile, the samples were slowly heated to $120{ }^{\circ} \mathrm{C}$, at which point the magnetic field was switched on and ramped to $9 \mathrm{~T}$ while the temperature continued to increase to $140^{\circ} \mathrm{C}$, well beyond the clearing temperatures of the smectic $\mathrm{A}^{*}$ mesophases but below the samples' order-to-disorder transition $\left(\sim 170{ }^{\circ} \mathrm{C}\right)$. The chamber was then allowed to cool at a rate of $1{ }^{\circ} \mathrm{C} / \mathrm{min}$ to $117{ }^{\circ} \mathrm{C}$ followed by a $15 \mathrm{~h}, 9 \mathrm{~T}$ dwell at that temperature. Under the second profile, the PS-PILC samples were exposed to the field during their disorder-order transitions by allowing the chamber to cool slowly from $T>T_{\mathrm{ODT}}$ into the $\mathrm{LC}$ regimes. The sample chamber was first heated to $180{ }^{\circ} \mathrm{C}\left(T_{\text {ODT }} \sim 170{ }^{\circ} \mathrm{C}\right.$ for both polymers) before the field was switched on. Heating continued to $200^{\circ} \mathrm{C}$ at $2{ }^{\circ} \mathrm{C} / \mathrm{min}$ followed by cooling to $180{ }^{\circ} \mathrm{C}$ at $1{ }^{\circ} \mathrm{C} / \mathrm{min}$, by which time the field had reached it maximum strength of $9 \mathrm{~T}$. This was followed by slow $0.1{ }^{\circ} \mathrm{C} / \mathrm{min}$ cooling to $160{ }^{\circ} \mathrm{C}$, into the microphase-separated regime, and further controlled cooling into the LC regime at $119{ }^{\circ} \mathrm{C}$. Both LCBCP samples were annealed at this temperature under the field for $90 \mathrm{~h}$. The temperature-field time profiles are given schematically in Figure 2. Temperature control was within $0.2{ }^{\circ} \mathrm{C}$. In both cases, at the end of the experiment, the magnetic field was completely ramped down before the samples were cooled to room temperature at $10^{\circ} \mathrm{C} /$ min. TEM and SAXS were then used for morphological characterization at room temperature. For SAXS, both a rotating copper anode $(\lambda=1.540 \AA)$ and a synchrotron source $(\lambda=1.307 \AA$ ) were used. Incident beam diameters were 100 and $400 \mu \mathrm{m}$ with abilities to resolve down to scattering vectors $q_{\text {min }}$ of 0.015 and $0.008 \AA^{-1}$, respectively.

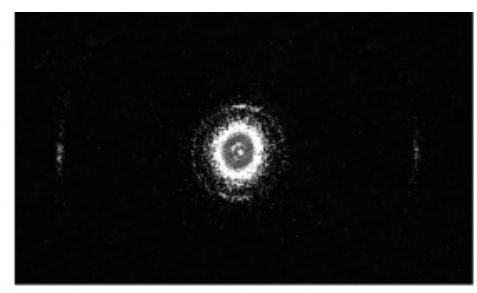

(a)

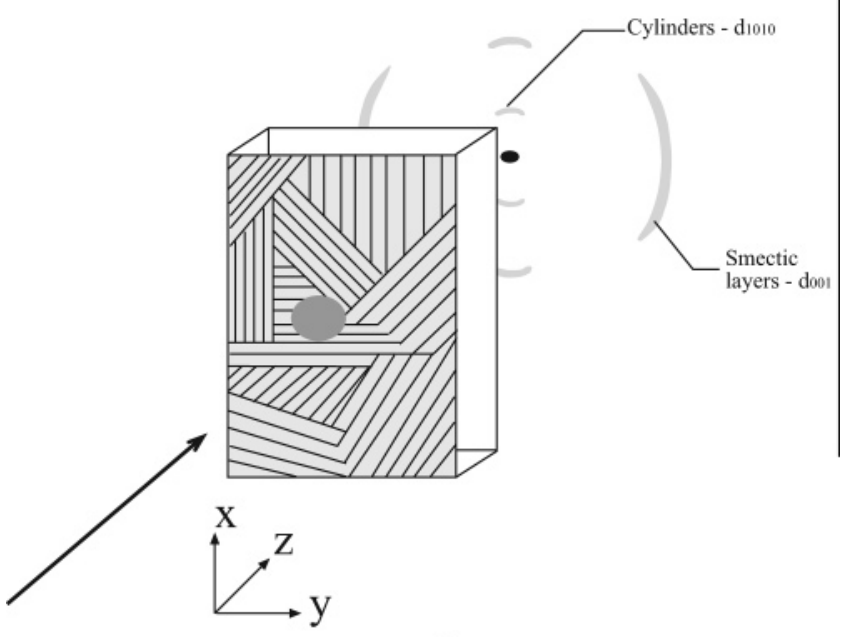

(b)

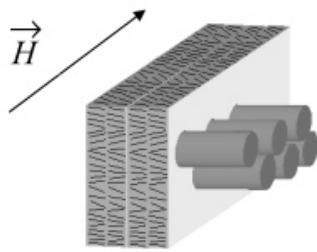

(c)

Figure 3. (a) Raw SAXS data recorded using a $100 \mu \mathrm{m}$ beam from a rotating anode source. The perpendicular orientation of the smectic layers with respect to the cylindrical microdomains is evident. (b) Schematic representation of SAXS data recorded using a $100 \mu \mathrm{m}$ beam from a rotating anode source. $\mathrm{X}$-rays are incident as indicated, along the $z$-axis, and probe the shaded area of material. The magnetic field used to orient the sample was also along the $z$-direction. The orthogonal orientation of the scattering from the smectic layers with respect to the hexagonally packed cylinder planes indicates that the mesogens are parallel to the IMDS. The cylinder long axes are, in turn, perpendicular to the magnetic field direction as indicated. (c) Schematic representation of the arrangement of smectic layers with respect to the hexagonally packed cylinders, indicating homogeneous anchoring of the mesogens at the cylinder IMDS.

\section{Results and Discussion}

No appreciable differences were found in the results obtained from the different temperature field profiles employed for each sample. Using the rotating anode SAXS with a $100 \mu \mathrm{m}$ beam, neither the PS-PILC 8K9K nor the PS-PB-PS samples showed any evidence of alignment of their microstructures, whereas in the PS-PILC $8 \mathrm{~K}-13 \mathrm{~K}$ sample, there was concentration of the small-angle X-ray scattering along arcs, indicating that alignment of the microdomains and the smectic layers in the matrix had taken place. The data are represented in raw form and, for clarity, schematically, in Figure $3 a-c$. From these data, it is inferred that the 


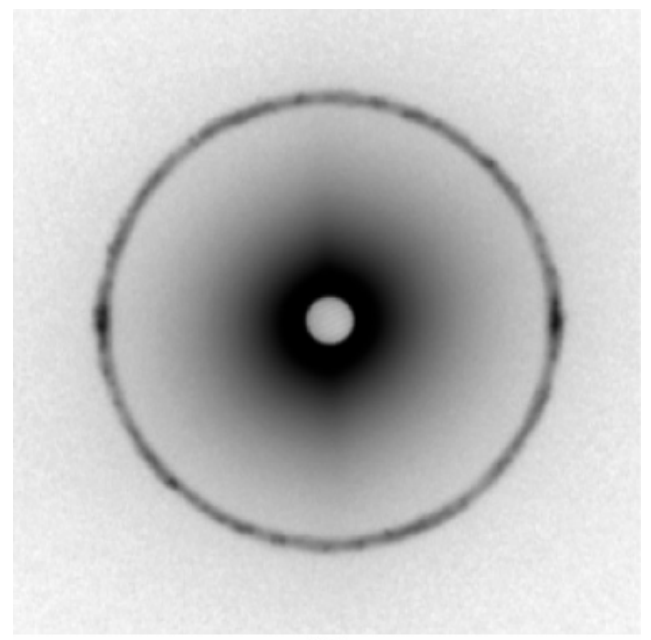

(a)

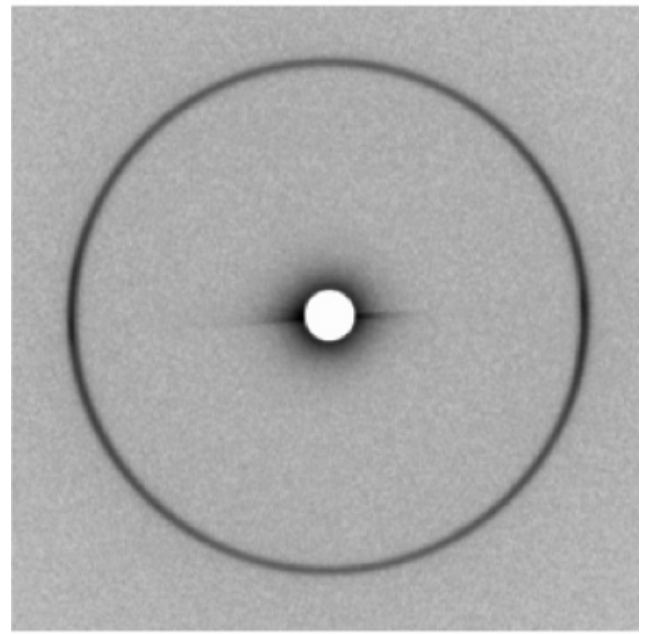

(b)

Figure 4. SAXS data recorded using a $400 \mu \mathrm{m}$, synchrotron beam. (a) Data from PS-PILC 8K-13K. (b) Data from PSPILC 8K-9K.

cylinder long axes and smectic layers align perpendicular to the field direction as shown in the schematic. Inspection of different parts of the sample produced scattering at different azimuthal locations but the relative orientation of the smectic arc to the hexagonally packed cylinder reflections was always orthogonal.

Scattering data taken using the bright, $400 \mu \mathrm{m}$ synchrotron derived beam also showed no distinguishable alignment for the PS-PILC 8K-9K and PS-PBPS samples, but for PS-PILC $8 \mathrm{~K}-13 \mathrm{~K}$, the data was typical of that of a polycrystalline sample. That is, there was a relatively discrete distribution of spot like reflections from the microdomains along the $q$ vector corresponding to the (1010) contribution of the hexagonal lattice, as shown in Figure 4. Hence, the sample consists of fairly large grains containing microdomains uniformly aligned perpendicular to the magnetic field. This is well supported by the results from TEM. The cylindrical microdomains were uniformly well-oriented perpendicular to the field in grains of approximately $20 \mu \mathrm{m}$ and larger, with the PS cylinders generally continuous across the grains. Representative data are shown in Figure 5. The integrated 2D SAXS data of Figure 4a are shown in Figure 6 and give $q$-vector ratios which confirm that the material is still composed of hexagonally ordered cylinders, rather than lamellae as one

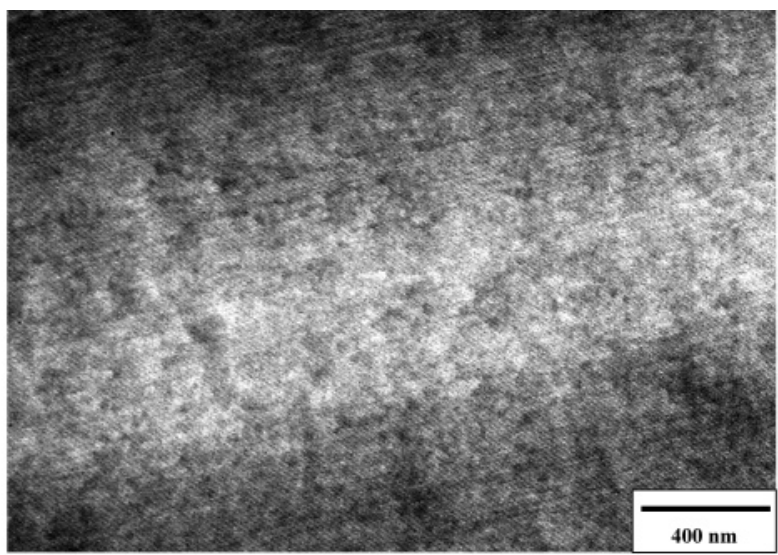

(a)

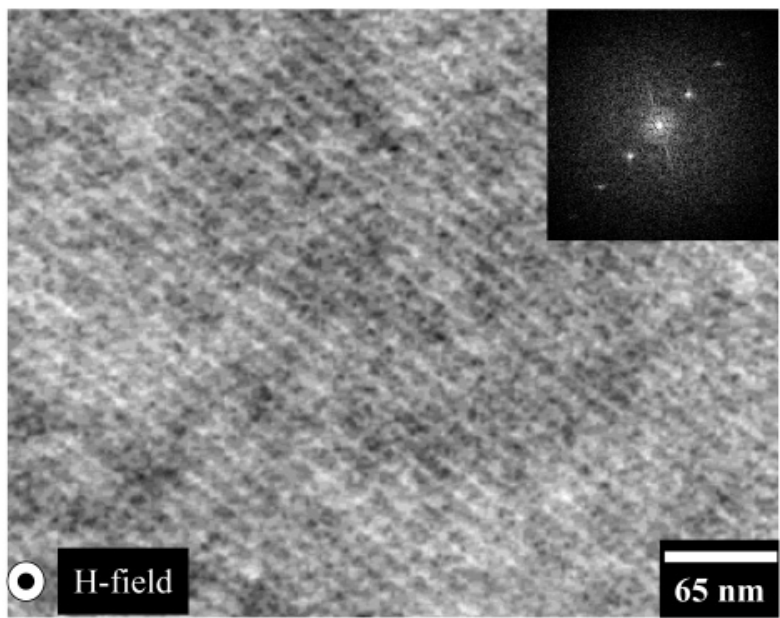

(b)

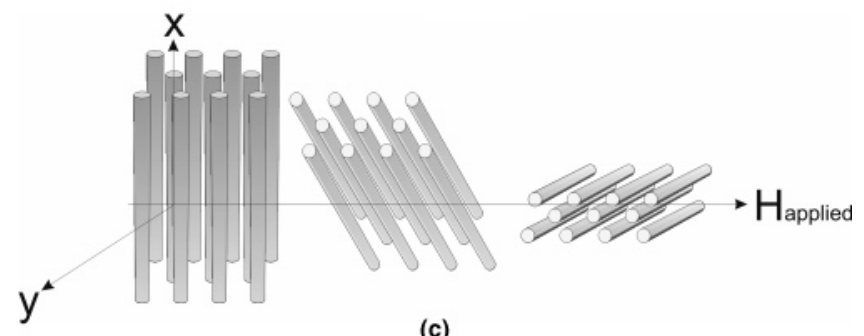

(c)

Figure 5. (a) TEM data from aligned polymer PS-PILC 8K$13 \mathrm{~K}$. The poly(styrene) cylinders have been stained using $\mathrm{RuO}_{4}$ vapors and thus appear dark. (b) A view of the same data at higher magnification. The inset Fourier transform confirms the excellent uniformity in the orientation of the cylinders. (c) Schematic of orientation of poly(styrene) cylinders relative to the homogeneously anchored LC mesogens in the presence of the magnetic field. The degeneracy in the morphology allows for all orientations in which the mesogen and cylinder long axes are contained in the $x-y$ plane.

might conclude from a casual inspection of the TEM data.

In general, observation of either parallel or perpendicular mesophase alignment with respect to magnetic field directions may be taken as indirect though conclusive evidence of positive and negative diamagnetic anisotropy. ${ }^{27}$ In the liquid crystalline diblock copolymers studied, there are contributions to anisotropy in the diamagnetic susceptibility from the microdomains themselves as well as from the LC mesophases within those microdomains. The form contribution from the microdomains is not expected to be significant, and this is borne out by the lack of any orientation evidenced in 


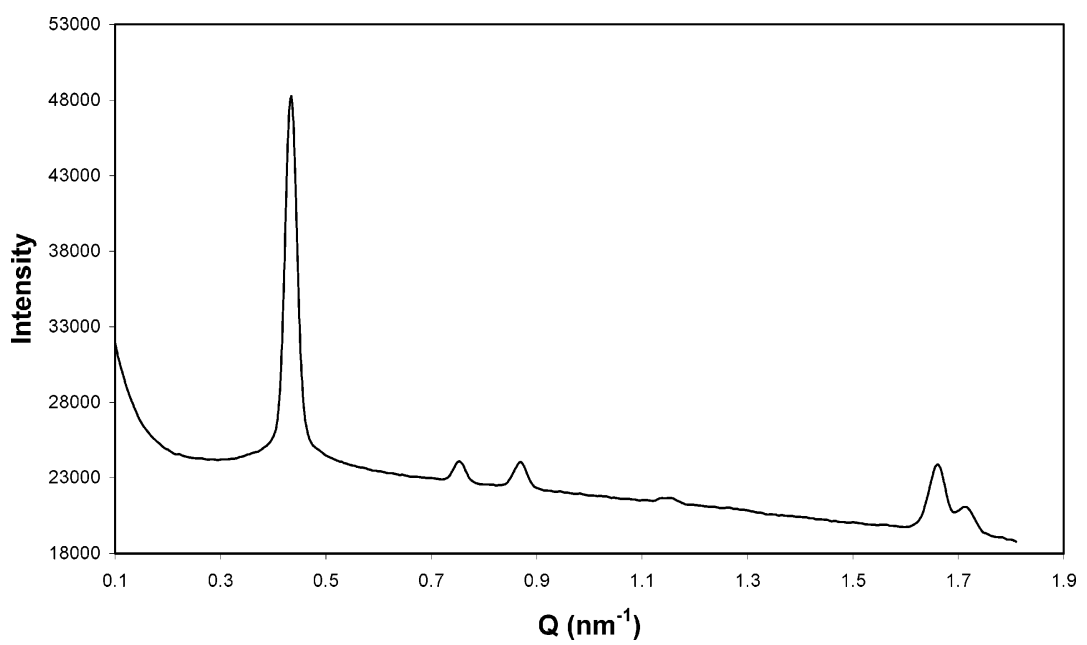

Figure 6. Scattered intensity vs scattering vector derived by circular integration of the SAXS data of Figure 4a. For the microdomains: $d^{0}=14.5 \mathrm{~nm}, d^{\prime}=8.4 \mathrm{~nm}, d^{\prime \prime} 7.3 \mathrm{~nm}, d^{\prime \prime}=5.5 \mathrm{~nm}$. The ratios of the $q$ vectors, 1:1.73:2:2.66, i.e., approximately $\sqrt{1}: \sqrt{3}: \sqrt{4}: \sqrt{7}$ indicate that the sample is composed of hexagonally packed cylinders, despite the lamellar appearance given by the uniform in plane orientation of the cylinder long axes in the TEM. For the LC mesophase: $d^{0}=3.8 \mathrm{~nm}$ and $d^{\prime}=3.7 \mathrm{~nm}$, in agreement with previously reported values. ${ }^{34}$

the PS-PB-PS coil-coil block copolymer. From the SAXS and TEM data, one can infer with confidence that the mesophase studied exhibits a negative diamagnetic anisotropy, given the perpendicular orientation of the smectic layers to the field direction. Studies were not carried out on the LC homopolymer, poly(isoprene-LC), as sufficient material was not available for experiments.

The alignment and large grain size produced in the cylinder forming PS-PILC sample are encouraging. The lack of alignment in the lamellar sample is well understood in terms of the relative orientation of the smectic layers to the microdomains in these materials. This orientation is governed by the planar or homogeneous anchoring condition of the mesogens at the IMDS. Planar anchoring at a flat surface, such as a lamellar IMDS, is degenerate as there are an infinite number of directions within the plane that the mesogen director can assume while still satisfying the requirement that it lie parallel to the surface. This is in stark contrast to planar anchoring at a singularly curved surface (i.e., cylinder IMDS) where there is one unique orientation in which the planar boundary condition is satisfiedthe mesogen long axis must lie parallel to the cylinder long axis. Thus, the requirement from the field that the mesophase lie perpendicular to the field lines imposes no unique orientation on the lamellar LCBCP whereas in the case of the cylinder forming LCBCP, the LC containing cylinders are forced to lie perpendicular to the field. These results are in excellent agreement with data garnered from oscillatory shear alignment of a related series of materials. In that study, ${ }^{28}$ liquid crystalline block copolymers based on higher molecular weight poly(styrene)-block-poly(isoprene) backbones functionalized on the isoprene block by an azobenzene-based mesogen were subjected to oscillatory shear under various conditions. The homogeneous anchoring condition of the mesogens at the IMDS resulted in the adoption of a unique transverse cylinder orientation with respect to the shear flow in the presence of the LC mesophase and a transition to a parallel cylinder orientation upon thermal isotropization of the LC phase while subjected to oscillatory shear. For the lamellar samples in that study, as in the present work, no unique microstructure orientations were observed. The anchoring condition and degeneracy at the planar lamellar
IMDS had implications for the thermal properties of the system as well-the final LC clearing temperatures of lamellar samples was significantly lower than those for cylinder forming samples, independent of molecular weight. $^{29}$

The negative diamagnetic anisotropy of the mesophase in the LCBCP system studied resulted in degeneracy in the orientation of the hexagonally packed cylinder microdomain structure with respect to the magnetic field lines. Clearly, there are an infinite number of in-plane orientations in which the cylinder long axes can lie perpendicular to the magnetic field direction. Given a positive diamagnetic anisotropy, one would expect that the cylinders likely would orient parallel to the field lines, yielding a sample with uniaxial symmetry, containing lateral cylinder axis invariant grain boundaries, ${ }^{30}$ i.e., directions normal to the grain boundary planes are parallel to the axis of the cylinders. Kinetics of grain growth and reorientation are mature areas of study in materials science, in fields such as ceramics and metallurgy. ${ }^{31}$ One can reasonably expect to apply some of the methods used in those fields to characterize the orientation process in the LCBCP materials studied here. The hurdle to creating truly single crystals of block copolymers can be alleviated by our ability to manipulate the order-disorder transition in these materials. It is generally well understood that to obtain high degrees of alignment over large areas, orienting forces should be applied when and where microstructural order is incipient, so that the final morphology is well influenced by the presence of the orienting force from inception. For example, careful thermal gradients at the solidification temperature of inorganics can be used to generate single crystals, and the procedure has been neatly mimicked in a careful work with block copolymers as well. ${ }^{32}$ In these LC systems, one would make the order-disorder transition and the liquid crystallization events concurrent to improve control over the orientation. This can be achieved using low molar mass block copolymers or weakly segregating blocks in which microphase separation is driven expressly by the liquid crystallization event. ${ }^{24,33}$ Passage through the disorder-order transition under an applied magnetic field would then give near single crystal texture sample, given an appropriate 
coupling of IMDS anchoring condition and diamagnetic anisotropy. The low molar mass also mitigates the viscous drag that works kinetically against grain reorientation post-microphase separation.

\section{Conclusions}

We have demonstrated the use of a magnetic field to orient a liquid crystalline diblock copolymer with a negative diamagnetic anisotropy. The results obtained agree very well with the oriented morphology obtained from oscillatory shear experiments on related diblocks, underscoring the importance of the anchoring condition of the mesogens with respect to the microdomain IMDS for determining the orientation of the superstructure. A basic set of specifications has been proposed for a LCBCP that can be oriented in a magnetic field such that the liquid crystalline block containing cylinders lie parallel to the field, allowing for the production of thin films of vertical or "standing" cylinders. There is significant room for extension of this work, including grain growth kinetic studies. The quality of alignment of the smectic layers as a function of annealing time and temperature can also be investigated more carefully and quantitatively by extracting the order parameters via small- and wide-angle X-ray scattering. This work contributes to the growing interest in magnetically active organics and organic-magnetic-inorganic hybrid materials.

Acknowledgment. We thank Dr. Yuanming Zhang of Cornell University and Professor Yachin Cohen of the Technion, Israel, for useful discussions and Professor B. Hsiao and Dr. F. Yeh of SUNY-Stony Brook for facilitating measurements at beamline X27C at Brookhaven. Funding was provided by the National Science Foundation through Grant DMR ECS-0103297 and by the Air Force Office of Scientific Research through MURI Grant F49620-97-1-0014.

\section{References and Notes}

(1) Walton, D. G.; Kellogg, G. J.; Mayes, A. M.; Lambooy, P.; Russell, T. P. Macromolecules 1994, 27, 6225-6228.

(2) Mayes, A. M.; Russell, T. P.; Bassereau, P.; Baker, S. M.; Smith, G. S. Macromolecules 1994, 27, 749-755.

(3) Russell, T. P.; Lambooy, P.; Kellogg, G. J.; Mayes, A. M. Physica B 1995, 213, 22-25.

(4) Segalman, R. A.; Yokoyama, H.; Kramer, E. J. Adv. Mater. 2001, 13, 1152.

(5) Park, C.; Yoon, J.; Thomas, E. L. Polymer 2003, 44, 67256760 .

(6) Fischer, H.; Poser, S.; Arnold, M.; Frank, W. Macromolecules 1994, $27,7133-7138$.
(7) Moment, A.; Hammond, P. T. Polymer 2001, 42, 6945-6959.

(8) Anthamatten, M.; Hammond, P. T. J. Polym. Sci., Part B: Polym. Phys. 2001, 39, 2671-2691.

(9) Itoh, T.; Yamada, M.; Hirao, A.; Nakahama, S. I.; Watanabe, J. Mol. Cryst. Liq. Cryst. 2000, 347, 455-464.

(10) Adams, J.; Sanger, J.; Tefehne, C.; Gronski, W. Macromol. Rapid Commun. 1994, 15, 879-886.

(11) Sanger, J.; Gronski, W.; Leist, H.; Wiesner, U. Macromolecules 1997, 30, 7621-7623.

(12) Zheng, W. Y.; Albalak, R. J.; Hammond, P. T. Macromolecules 1998, 31, 2686-2689.

(13) Manthis, A.; Hadziioannou, G.; Skoulios, A. Polym. Eng. Sci. 1977, 17, 570-572.

(14) Keller, A.; Pedemonte, E.; Willmouth, F. M. Nature (London) 1970, 225 .

(15) Morrison, F. A.; Winter, H. H. Macromolecules 1989, 22, $3533-3540$.

(16) Thurn-Albrecht, T.; DeRouchey, J.; Russell, T. P.; Jaeger, H. M. Macromolecules 2000, 33, 3250-3253.

(17) De Rosa, C.; Park, C.; Lotz, B.; Wittmann, J. C.; Fetters, L. J.; Thomas, E. L. Macromolecules 2000, 33, 4871-4876.

(18) Park, C.; De Rosa, C.; Fetters, L. J.; Lotz, B.; Thomas, E. L. Adv. Mater. 2001, 13, 724-728.

(19) De Rosa, C.; Park, C.; Thomas, E. L.; Lotz, B. Nature (London) 2000, 405, 433-437.

(20) Park, C.; De Rosa, C.; Thomas, E. L. Macromolecules 2001, $34,2602-2606$

(21) Wittmann, J. C.; Meyer, S.; Damman, P.; Dosiere, M.; Schmidt, H. W. Polymer 1998, 39, 3545-3550.

(22) Fenwick, D.; Smith, P.; Wittmann, J. C. J. Mater. Sci. 1996, $31,128-131$.

(23) Thierry, A.; Mathieu, C.; Straupe, C.; Wittmann, J. C.; Lotz, B. Macromol. Symp. 2001, 166, 43-58.

(24) Hamley, I. W.; Castelletto, V.; Lu, Z. B.; Imrie, C. T.; Itoh, T.; Al-Hussein, M. Macromolecules 2004, 37, 4798-4807.

(25) Mao, G. P.; Wang, J. G.; Ober, C. K.; Brehmer, M.; O’Rourke, M. J.; Thomas, E. L. Chem. Mater. 1998, 10, 1538-1545.

(26) Mao, G. P.; Wang, J. G.; Clingman, S. R.; Ober, C. K.; Chen, J. T.; Thomas, E. L. Macromolecules 1997, 30, 2556-2567.

(27) Galli, G.; Chiellini, E.; Francescangeli, O.; Ferri, D.; Wolff, D.; Springer, J.; Laus, M.; Angeloni, A. S. Macromol. Symp. 1997, 121, 235-244.

(28) Osuji, C.; Zhang, Y. M.; Mao, G. P.; Ober, C. K.; Thomas, E. L. Macromolecules 1999, 32, 7703-7706.

(29) Osuji, C. O.; Chen, J. T.; Mao, G.; Ober, C. K.; Thomas, E. L. Polymer 2000, 41, 8897-8907.

(30) Martin, D. C.; Thomas, E. L. Philos. Mag. A 1991, 64, 903922.

(31) Ferreira, P. J.; Liu, H. B.; Vander Sande, J. B. J. Mater. Res. 1999, 14, 2751-2763.

(32) Hashimoto, T.; Bodycomb, J.; Funaki, Y.; Kimishima, K. Macromolecules 1999, 32, 952-954.

(33) Anthamatten, M.; Zheng, W. Y.; Hammond, P. T. Macromolecules 1999, 32, 4838-4848.

(34) Mao, G. P. Molecular Design, Synthesis, Characterization and Processing of Liquid Crystalline Diblock Copolymers. Ph.D. Thesis, Cornell University, 1997.

MA0483064 